\title{
SOME THEOREMS ON BOUNDED ANALYTIC FUNCTIONS
}

\author{
BY \\ WALTER RUDIN
}

1. Introduction. In a paper by Bers [3] the following unpublished theorem of Chevalley and Kakutani is mentioned in a footnote:

Theorem A. If to each boundary point $W$ of a domain $B$ there exists a bounded analytic function defined in $B$ and possessing at $W$ a singularity, then $B$ is determined (modulo a conformal transformation) by the ring of all bounded analytic functions on $B$.

The purpose of this paper is twofold. A proof of Theorem A is given which, according to Professor Kakutani, is considerably simpler than the original one (Theorem 9; a slight extension is stated in Theorem 12). In particular, no use is made of the theory of normed rings and of the topology of the maximal ideal space; our methods are more function-theoretic. Secondly, we investigate those properties of the boundary of a domain which are connected with the existence of singularities of bounded analytic functions. This leads to the consideration of two classes of boundary points (removable and essential) and of their relation to Painlevé null-sets (Theorem 5). A theorem on cluster sets is established (Theorem 14), and it is shown (Theorem 15) that the domains considered by Chevalley and Kakutani (we shall call these domains maximal) are precisely those which are domains of existence of single-valued bounded analytic functions, i.e., those whose boundaries are natural boundaries of some such function. Theorem 11 shows that every domain $D$ is contained in a unique smallest maximal domain $D^{*}$.

2. Definitions. Let $B(D)$ denote the set of all single-valued bounded analytic functions on the domain $D$ (by a domain we mean a connected open subset of the Riemann sphere $S$; unless the contrary is stated, we shall always assume that the boundary of $D$ is bounded; this involves no loss of generality, since all our results are invariant under conformal one-to-one mappings of $S$ onto $S$ ).

Let $f \in B(D)$. If $D_{1}$ is a domain such that $D_{1} \cap D$ is not vacuous, and if there is a function $f_{1} \in B\left(D_{1}\right)$ such that $f(z)=f_{1}(z)$ for $z \in D_{1} \cap D$, we say that $f$ can be extended to $D_{1}$.

A boundary point $x$ of $D$ is said to be removable if for every $f \in B(D)$ there exists a neighborhood $V$ of $x$ such that $f$ can be extended to $V$. By an essential boundary point of $D$ we mean one that is not removable. If every boundary

Presented to the Society, December 29, 1953, under the titles Natural boundaries of bounded analytic functions and Rings of bounded analytic functions; received by the editors January 7, 1954. 
point of $D$ is essential, we say that $D$ is maximal.

The closed disc with center at $x$ and radius $r$ will be denoted by $J(x, r)$.

3. Painlevé null-sets. Let $K$ be the complement of a domain $D$. If $B(D)$ consists of the constant functions alone, then $K$ is called a Painleve null-set $[2$, p. 107] or simply a null-set. It is known that $K$ is a null-set if the linear measure of $K$ is zero [1, p. 2]; if $K$ is a subset of an analytic arc, this sufficient condition is also necessary $[2$, p. 122$]$; in the general case, no characterization of this sort has yet been found.

It is trivial that null-sets are totally disconnected, and it is easily shown [2, p. 108] that $K$ is a null-set if and only if every $f \in B(\Delta-K)$ can be extended to $\Delta$, where $\Delta$ is any domain containing $K$. This remark leads to a simple proof of the following property, which we state as a lemma:

4. Lemma. Let $\Delta$ be a domain, and let $K$ be a closed set. Suppose that for every $x \in \Delta \cap K$ there exists $r>0$ ( $r$ depending on $x$ ) such that $J(x, r) \subset \Delta$ and $J(x, r) \cap K$ is a Painlevé null-set. Then every $f \in B(\Delta-K)$ can be extended to $\Delta$.

Next we show that if $x$ is a removable boundary point of $D$, there exists a domain $D_{1}$ containing $x$ and $D$, independent of $f$, such that every $f \in B(D)$ can be extended to $D_{1}$ :

5. Theorem. Let $K$ be the complement of the domain D. A point $x \in K$ is a removable boundary point of $D$ if and only if the set $K \cap J(x, r)$ is a Painlevé null-set for some $r>0$.

Proof. The sufficiency of the condition is evident. To prove the necessity, suppose $K \cap J(x, r)$ is not a null-set for any $r>0$. Let $V$ be a neighborhood of $x$, and put $\Delta=V-\{x\}$. If for every $y \in \Delta$ there exists an $r>0$ such that $K \cap J(y, r)$ is a null-set, then every $f \in B(\Delta-K)$ can be extended to $\Delta$, by Lemma 4 , and hence to $V$, so that $K \cap J(x, r)$ is a null-set provided $J(x, r) \subset V$.

This contradiction shows that there exists a sequence of disjoint discs $J\left(x_{n}, r_{n}\right) \subset \Delta(n=1,2,3, \cdots)$ such that $x_{n} \rightarrow x$ and such that $K_{n}=K \cap J\left(x_{n}, r_{n}\right)$ is not a null-set. The complement $D_{n}$ of $K_{n}$ contains $D$ and is connected (being the union of two connected sets). Choose nonconstant functions $f_{n} \in B\left(D_{n}\right)$ such that $\left|f_{n}(z)\right|<2^{-n}$ in $D_{n}$, and define $f(z)=\sum f_{n}(z)$.

Then $f \in B(D)$. Since $f=\left(f-f_{n}\right)+f_{n}$ and $f-f_{n}$ can be extended to a neighborhood of $K_{n}$, whereas this is not true of $f_{n}$, we see that $f$ cannot be extended to any neighborhood of $x$. Hence $x$ is an essential boundary point of $D$, and the theorem follows.

The following two lemmas will be needed in the proof of Theorem 9 .

6. Lemma. Suppose $\Delta$ is an open set which is dense in a domain D. If $\phi$ is analytic in $D$ (with possible poles) and schlicht in $\Delta$, then $\phi$ is schlicht in $D$.

Proof. Suppose there are two distinct points $z_{1}, z_{2}$ in $D$ such that $\phi\left(z_{1}\right)$ 
$=\phi\left(z_{2}\right)$, and let $V_{1}, V_{2}$ be disjoint neighborhoods of $z_{1}, z_{2}$, such that $V_{1} \subset D$. $V_{2} \subset D$.

Since $\phi\left(V_{i} \cap \Delta\right)$ is an open set, dense in $\phi\left(V_{i}\right)(i=1,2)$, we see that $\phi\left(V_{1} \cap \Delta\right)$ and $\phi\left(V_{2} \cap \Delta\right)$ have a point in common; this point has two distinct inverse images in $\Delta$, a contradiction.

7. Lemma. Let $D_{1}$ be a domain. If $D_{1}$ is maximal and conformally equivalent to $D_{2}$, then $D_{2}$ is also a maximal domain.

Proof. Suppose $\phi$ maps $D_{2}$ conformally onto $D_{1}$. We assume, without loss of generality, that $\phi$ is analytic, rather than conjugate analytic. If $D_{2}$ has a removable boundary point $x$, there exists a neighborhood $V$ of $x$ such that every $f \in B\left(D_{2}\right)$ can be extended to $D_{2}^{\prime}=V \cup D_{2}$, by Theorem 5 and Lemma 4 . $D_{2}$ is clearly dense in $D_{2}^{\prime}$, and since $\phi$ is bounded except in the neighborhood of the point $z_{0} \in D$ such that $\phi\left(z_{0}\right)=\infty$ (if $D_{1}$ contains the point at infinity), $\phi$ can be extended to $D_{2}^{\prime}$. By Lemma $6, \phi$ is schlicht in $D_{2}^{\prime}$, so that the domain $D_{1}^{\prime}=\phi\left(D_{2}^{\prime}\right)$ contains $D_{1}$ properly.

Choose $f \in B\left(D_{1}\right)$, and set $g(w)=f(\phi(w))$ for $w \in D_{2}$. Since $g \in B\left(D_{2}\right), g$ can be extended to $D_{2}^{\prime}$, and the formula: $f(z)=g\left(\phi^{-1}(z)\right)$ extends $f$ to $D_{1}^{\prime}$, contradicting the maximality of $D$.

Hence $D_{2}$ has no removable boundary point.

8. Before turning to the proof of the theorem of Chevalley and Kakutani, we insert the following remark: We shall consider two domains, $D_{1}$ and $D_{2}$, and an isomorphic mapping $\psi$ of the ring $B\left(D_{1}\right)$ onto the ring $B\left(D_{2}\right)$, i.e., a one-to-one mapping which preserves sums and products; it is clear that $\psi(1)=1$. Since the imaginary unit $i$ is a primitive fourth root of unity, we must either have $\psi(i)=i$ or $\psi(i)=-i$. In the former case, we call $\psi$ a direct isomorphism. If $\psi(i)=-i$, conjugates must be introduced into (1) below in an obvious manner, and the induced correspondence between the points of $D_{1}$ and $D_{2}$, while still conformal, reverses orientation.

9. Theorem. Let $D_{1}$ and $D_{2}$ be maximal domains which are proper subsets of the Riemann sphere, and let $\psi$ be a direct isomorphism mapping $B\left(D_{1}\right)$ onto $B\left(D_{2}\right)$. Then there exists a one-to-one conformal mapping $\phi$ of $D_{2}$ onto $D_{1}$ such that

$$
f(\phi(w))=f^{*}(w) \quad\left(w \in D_{2}\right),
$$

where $f^{*}=\psi(f)$.

Since the converse of the theorem is obvious, we can summarize the situation by saying that $D_{1}$ and $D_{2}$ are conformally equivalent if and only if $B\left(D_{1}\right)$ and $B\left(D_{2}\right)$ are algebraically isomorphic.

Proof. We assume, without loss of generality, that $D_{1}$ and $D_{2}$ are in the finite plane. 
Using the notation $f^{*}=\psi(f)$, we readily see that $r^{*}=r$ for any rational number $r$ (i.e., for any constant function whose range is the number $r$ ). Having assumed that $i^{*}=i$, we see that $r^{*}=r$ for any complex rational $r$ (by a complex rational we mean a complex number with rational real and imaginary parts).

Let $Q(f)$ denote the closure of the range of $f$. For any $f \in B\left(D_{1}\right)$ and any complex rational $r$, the following four statements are clearly equivalent, since

$$
(f-r)^{*}=f^{*}-r^{*}=f^{*}-r
$$

and $\psi$ preserves reciprocals:

(a) $r \in Q(f)$.

(b) $f-r$ has no reciprocal in $B\left(D_{1}\right)$.

(c) $f^{*}-r$ has no reciprocal in $B\left(D_{2}\right)$.

(d) $r \in Q\left(f^{*}\right)$.

Thus $Q(f)$ and $Q\left(f^{*}\right)$ contain the same complex rationals.

It follows that for any constant $c, c^{*}$ is also a constant. Furthermore, if $f$ is not constant, then the same is true of $f^{*}$; since $Q(f)$ and $Q\left(f^{*}\right)$ are closures of open sets, we conclude that

$$
Q(f)=Q\left(f^{*}\right) \quad\left(f \in B\left(D_{1}\right), f \neq \text { const. }\right) .
$$

Now let $c$ be any constant, choose a nonconstant $f \in B\left(D_{1}\right)$ such that $0 \in Q(f)$, and put

$$
f_{n}(z)=c+f(z) / n \quad\left(z \in D_{1}, n=1,2,3, \cdots\right) .
$$

Then

$$
f_{n}^{*}(w)=c^{*}+f^{*}(w) / n \quad\left(w \in D_{2}, n=1,2,3, \cdots\right),
$$

and $c \in Q\left(f_{n}\right), c^{*} \in Q\left(f_{n}^{*}\right)$. By (2) we have

$$
c=\bigcap_{n=1}^{\infty} Q\left(f_{n}\right)=\bigcap_{n=1}^{\infty} Q\left(f_{n}^{*}\right)=c^{*},
$$

so that $c^{*}=c$ for every constant $c$.

Now, if $D_{1}$ is unbounded, the identity function $I(z)=z$ is not a member of $B\left(D_{1}\right)$. This difficulty cannot, in general, be eliminated by mapping $D_{1}$ conformally onto a bounded domain: Ahlfors and Beurling [2] have shown that there are domains $D$ on which there exist no bounded schlicht functions, although $B(D)$ contains nonconstant functions; such a domain $D$ is not conformally equivalent to any bounded domain.

However, let $F\left(D_{j}\right)$ be the quotient field of $B\left(D_{j}\right)(j=1,2)$, and extend $\psi$ in the natural manner to an isomorphic mapping of $F\left(D_{1}\right)$ onto $F\left(D_{2}\right)$. We shall see that $I \in F\left(D_{1}\right)$ and that the function $\phi=\psi(I) \in F\left(D_{2}\right)$ is the desired mapping function for which (1) holds. 
For any nonconstant $f \in B\left(D_{1}\right)$ and any point $a \in D_{1}$, put

$$
g(z)=(f(z)-f(a)) /(z-a)
$$

so that

$$
I(z)=z=a+(f(z)-f(a)) / g(z) .
$$

These formulas show that $g \in B\left(D_{1}\right)$ and hence that $I \in F\left(D_{1}\right)$.

Now set $\phi=\psi(I), \phi\left(D_{2}\right)=D_{3}, D=D_{1} \cap D_{3}$. For any constant $c$, the following four statements are clearly equivalent:

(a) $c \in Q(I)$.

(b) The reciprocal of $I-c$ is not in $B\left(D_{1}\right)$.

(c) The reciprocal of $\phi-c$ is not in $B\left(D_{2}\right)$.

(d) $c \in Q(\phi)$.

Thus $D_{1}$ and $D_{3}$ have the same closure; it follows that $D$ is dense in $D_{1}$ and in $D_{3}$.

We have to show that $\phi$ is schlicht, and that $D_{1}=D_{3}$.

Choose any point $a \in D$ and let $M_{a}$ be the set of all functions $f \in B\left(D_{1}\right)$ such that $f(a)=0 . M_{a}$ is evidently an ideal. If $g \in B\left(D_{1}\right)$ and $g(a) \neq 0$, then the identity

$$
1=\frac{g(a)-g(z)}{g(a)}+\frac{g(z)}{g(a)}
$$

shows that every ideal containing $M_{a}$ and $g$ contains the constant 1 and hence the whole ring $B\left(D_{1}\right)$. Thus $M_{a}$ is a maximal ideal of $B\left(D_{1}\right)$, which $\psi$ maps onto a corresponding maximal ideal $M_{a}^{*}$ of $B\left(D_{2}\right)$.

For every $f \in M_{a}, f /(I-a) \in B\left(D_{1}\right)$, so that $f^{*} /(\phi-a) \in B\left(D_{2}\right)$ for every $f^{*} \in M_{a}^{*}$. It follows that $f^{*}(w)=0$ whenever $\phi(w)=a$, for every $f^{*} \in M_{a}^{*}$. If there were more than one such point $w \in D_{2}$, the ideal $M_{a}^{*}$ would not be maximal. We conclude: for every $a \in D$, there exists precisely one point $b \in D_{2}$ such that $\phi(b)=a$.

If $\Delta$ is the set of all $b \in D_{2}$ such that $\phi(b) \in D$, the fact that $D$ is dense in $D_{3}$ implies that $\Delta$ is dense in $D_{2}$, since the analytic function $\phi$ is an open mapping. Lemma 6 now shows that $\phi$ is schlicht.

Thus $D_{3}$ is conformally equivalent to $D_{2}$; by Lemma $7, D_{3}$ is maximal.

To complete the proof, choose $f \in B\left(D_{1}\right), a \in D, b \in D_{2}$ such that $\phi(b)=a$. Then $f-f(a) \in M_{a}$, so that $f^{*}-f(a) \in M_{a}^{*}$. But $M_{a}^{*}$ consists of those functions of $B\left(D_{2}\right)$ which vanish at $b$. Thus $f^{*}(b)=f(a)$, or

$$
f(z)=f^{*}\left(\phi^{-1}(z)\right) \quad\left(f \in B\left(D_{1}\right), z \in D\right) .
$$

Since $\phi$ maps $D_{2}$ conformally onto $D_{3}$, there is a function $g \in B\left(D_{3}\right)$ such that

$$
g(z)=f^{*}\left(\phi^{-1}(z)\right)
$$


The functions $f$ and $g$ coincide in $D$; thus every $f \in B\left(D_{1}\right)$ can be extended to $D_{3}$.

On the other hand, for every $g \in B\left(D_{3}\right)$ there is an $f^{*} \in B\left(D_{2}\right)$ for which (4) holds; defining $f=\psi^{-1}\left(f^{*}\right)$, we obtain a member of $B\left(D_{1}\right)$ for which (3) holds; thus every $g=B\left(D_{3}\right)$ can be extended to $D_{1}$.

Since $D_{1}$ and $D_{3}$ are maximal domains, we conclude that $D_{1}=D_{3}$. The theorem follows.

10. Remark. Let us apply Theorem 9 to the case $D_{1}=D_{2}=D$, say. Let $\psi_{1}, \psi_{2}$ be two direct automorphisms of $B(D)$, and let $\phi_{1}, \phi_{2}$ be the corresponding conformal transformations of $D$ onto itself, in accordance with formulas (1). Then it is easily seen that the automorphism $\psi_{2} \psi_{1}$ corresponds to the mapping $\phi_{1} \phi_{2}$, i.e., the mapping taking $z$ into $\phi_{1}\left(\phi_{2}(z)\right)$. Replacing the automorphisms $\psi$ by their inverses, the following is evident:

For every maximal domain $D$, there is a natural isomorphism between the group of all direct automorphisms of the ring $B(D)$ and the group of all directly conformal one-to-one mappings of $D$ onto $D$.

We now return to our investigation of the properties of essential boundary points.

11. TheOREM. If $K^{*}$ is the set of all essential boundary points of a domain $D$, then $K^{*}$ is perfect (if not vacuous). If $D^{*}$ is that component of the complement of $K^{*}$ which contains $D$, then $D$ is dense in $D^{*}$, and $D^{*}$ is the smallest maximal domain which contains $D$.

Proof. Theorem 5 shows that $D^{*}$ may be obtained in the following manner: cover every removable boundary point $x$ of $D$ with an open disc $U_{x}$ which does not intersect $K^{*} ; D^{*}$ is the union of $D$ and these discs $U_{x}$. Since null-sets are totally disconnected, $D$ is dense in $D^{*}$.

By Lemma 4, every $f \in B(D)$ can be extended to $D^{*}$. Thus there is no smaller maximal domain containing $D$. To show that $D^{*}$ is maximal, let $x$ be a boundary point of $D^{*}$. Then $x$ is an essential boundary point of $D$, there is some $f \in B(D)$ which cannot be extended to any neighborhood of $x$, and the same is of course true of the extension of $f$ to $D^{*}$. Thus $x$ is an essential boundary point of $D^{*}$.

Thus $D^{*}$ is maximal, and since isolated boundary points are removable, $K^{*}$ is perfect (or vacuous).

As a consequence of Theorem 11, Theorem 9 may now be restated as follows:

12. Theorem. Let $D_{1}^{*}$ and $D_{2}^{*}$ be the smallest maximal domains containing the domains $D_{1}$ and $D_{2}$. If $B\left(D_{1}\right)$ and $B\left(D_{2}\right)$ are algebraically isomorphic, then $D_{1}^{*}$ and $D_{2}^{*}$ are conformally equivalent.

13. Definition. Let $x$ be a boundary point of a domain $D$, and let $f$ be analytic in $D$. Put $D_{n}=D \cap J(x, 1 / n)$, and let $E_{n}$ be the closure of $f\left(D_{n}\right)$ (the range of the restriction of $f$ to $D_{n}$ ). The set 


$$
C(f ; x)=\bigcap_{n=1}^{\infty} E_{n}
$$

is called the cluster set of $f$ at $x$.

It is clear that for every $w \in C(f ; x)$ there is a sequence $\left\{z_{n}\right\}$ such that $z_{n} \in D, z_{n} \rightarrow x$, and $f\left(z_{n}\right) \rightarrow w$ as $n \rightarrow \infty$.

If $x$ is an essential boundary point of $D$, then there is some $f \in B(D)$ with a singularity at $x$. The question as to how "bad" this singularity can be is partially answered by our next theorem; the example given at the end of this paper has its origin in the same question.

14. Theorem. Let $x$ be an essential boundary point of the domain $D$. There exists a function $f \in B(D)$ whose cluster set at $x$ consists of the entire closed unit disc, although $|f(z)|<1$ for every $z \in D$.

Proof. Since the set of essential boundary points of $D$ is perfect, there is a sequence $\left\{x_{n}\right\}$ of distinct essential boundary points such that $x_{n} \neq x$ and $x_{n} \rightarrow x$ as $n \rightarrow \infty$. Choose $\left\{r_{n}\right\}$ such that no two of the discs $T_{n}=J\left(x_{n}, r_{n}\right)$ intersect and such that $T_{n}$ contains no point of the interior $I$ of $K$ if $x_{n}$ is not a limit point of $I$. Let $K_{n}=K \cap J\left(x_{n}, r_{n} / 3\right)$, let $G_{n}$ be the complement of $K_{n}$, and put $d_{n}=D \cap J\left(x_{n}, r_{n} / 2\right)$. As usual, $K$ denotes the complement of $D$.

We shall construct an increasing sequence $\left\{n_{k}\right\}$ of positive integers, and functions $f_{k} \in B\left(G_{n_{k}}\right)(k=1,2,3, \cdots)$ such that

(i) $\left|f_{k}(z)\right|<1$ for $z \in G_{n_{k}}, f_{k}(x)=0$;

(ii) for some sequence $\left\{z_{i}\right\}$ of points in $d_{n_{k}}, f_{k}\left(z_{i}\right) \rightarrow 1$ as $i \rightarrow \infty$;

(iii) $\left|f_{k}(z)\right|<2^{-k-1}$ for $z \in G_{n_{k}}-T_{n_{k}}$;

(iv) $\sum_{i=1}^{k}\left|f_{i}(z)\right|<2^{-k-2}$ for $z \in T_{n_{k+1}}$.

If $x_{n}$ is not a limit point of $I$, choose a nonconstant $u_{n} \in B\left(G_{n}\right)$ such that $\sup \left|u_{n}(z)\right|=1$ for $z \in G_{n}$, hence also for $z \in d_{n}$, since $d_{n}$ is dense in $G_{n}$.

If $x_{n}$ is a limit point of $I$, choose $y_{n} \in I$ such that $\left|x_{n}-y_{n}\right|<r_{n} / 6$, let $p_{n}$ be the distance from $y_{n}$ to $D$, and set $u_{n}(z)=p_{n} /\left(z-y_{n}\right)$.

In either case, let us multiply $u_{n}$ by a suitable constant of modulus 1 so as to make $u_{n}(x)$ real, and let us define

$$
v_{n}(z)=\frac{u_{n}(z)-u_{n}(x)}{1-u_{n}(x) u_{n}(z)} .
$$

Then $v_{n} \in B\left(G_{n}\right),\left|v_{n}(z)\right|<1$ in $G_{n}$, and $v_{n}(x)=0$; multiplying $v_{n}$ by a suitable constant of modulus 1 , we may further arrange it so that $v_{n}\left(z_{i}\right) \rightarrow 1$ for some sequence $\left\{z_{i}\right\}$ in $d_{n}$.

Now take $n_{1}=1, g_{1}=v_{1}$. Having picked $n_{1}, \cdots, n_{k}$ and $g_{1}, \cdots, g_{k}$, take $n_{k+1}>n_{k}$ such that (iv) holds with $g_{i}$ in place of $f_{i}$ (this is possible since $g_{i}(x)$ $=0$ and $\left.x_{i} \rightarrow x\right)$, and put $g_{k+1}=v_{n_{k+1}}$.

Finally, let $f_{k}=\left(g_{k}\right)^{m_{k}}$, where $m_{k}$ is a positive integer chosen large enough for (iii) to hold. Then all of the conditions (i) to (iv) are satisfied. 
Next, let $E$ be a countable dense subset of the open unit disc, and let $\left\{w_{n}\right\}$ be a sequence of complex numbers which contains every member of $E$ infinitely many times, with the additional requirement that $\left|w_{n}\right|<1-2^{-n}$.

The function

$$
f(z)=\sum_{k=1}^{\infty} w_{k} f_{k}(z)
$$

has the desired properties. By (iii) and (iv) we have, for $z \in D \cap T_{n_{m}}$,

$$
\begin{aligned}
|f(z)| & <\sum_{1}^{m-1}\left|f_{k}(z)\right|+\left|w_{m}\right|+\sum_{m+1}^{\infty}\left|f_{k}(z)\right| \\
& <2^{-m-1}+1-2^{-m}+\sum_{m+1}^{\infty} 2^{-k-1}=1 .
\end{aligned}
$$

If $z \in D-T_{n_{m}}$ for every $m$, then $|f(z)|<\sum 2^{-k-1}=1 / 2$. Thus $|f(z)|<1$ for every $z \in D$.

Finally, choose $z \in d_{n_{m}}$ such that $\left|f_{m}(z)-1\right|<2^{-m}$. Then

$$
\left|f(z)-w_{m}\right|<\sum_{1}^{m-1}\left|f_{k}(z)\right|+\left|w_{m}\right| \cdot\left|f_{m}(z)-1\right|+\sum_{m+1}^{\infty}\left|f_{k}(z)\right|<2^{-m+1} \text {. }
$$

Thus every member of $E$ is in $C(f ; x)$; since $C(f ; x)$ is evidently closed, the theorem follows.

15. Theorem. If $D$ is a maximal domain, there exists a function $f \in B(D)$ which has the boundary of $D$ as its natural boundary.

Proof. Suppose, without loss of generality, that $D$ contains the point at infinity. Let $\left\{x_{n}\right\}$ be a countable dense subset of the boundary of $D$. The preceding theorem, applied to each $x_{n}$, shows that there exist functions $f_{n} \in B(D)$ and points $z_{n} \in D$ such that $\left|f_{n}(z)\right|<1$ in $D, f_{n}(\infty)=0,\left|x_{n}-z_{n}\right|<1 / n$, $f_{n}\left(z_{n}\right)=a_{n}$, where $a_{n}$ is real and

$$
\sum_{n=1}^{\infty}\left(1-a_{n}\right)<\infty
$$

Define

$$
f(z)=\prod_{n=1}^{\infty} \frac{a_{n}-f_{n}(z)}{1-a_{n} f_{n}(z)} .
$$

By a generalization of Schwarz's lemma, for every closed subset $C$ of $D$ there is a constant $M<1$ such that $\left|f_{n}(z)\right|<M$ on $C$. For $z \in C$, we have

$$
\left|1-\frac{a_{n}-f_{n}(z)}{1-a_{n} f_{n}(z)}\right|<\frac{1+M}{1-M}\left(1-a_{n}\right),
$$


so that the product defining $f(z)$ converges uniformly on $C$. Since $f\left(z_{n}\right)=0$ and every boundary point of $D$ is a limit point of the set $\left\{z_{n}\right\}$, the theorem follows.

16. An example. In view of Theorem 14 , one may ask the following question: given an essential boundary point $x$ of the domain $D$ and an arc $L$ which lies in $D$, except for one of its end points, which is at $x$, does there exist a nonconstant function $f \in B(D)$ such that (a) $f(z)$ does not converge as $z \rightarrow x$ along $L$, or, (b) such that $|f(z)|$ tends to its maximum as $z \rightarrow x$ along $L$ ?

It can be shown that if for some $x$ the answer to (b) is affirmative, the same is true for (a). We shall give an example in which the answers to both (a) and (b) are negative.

Let $\left\{a_{n}\right\}$ be a sequence of distinct points on the positive real axis, converging to zero. Choose $\left\{R_{n}\right\}$ such that the discs $J\left(a_{n}, R_{n}\right)$ are mutually disjoint, let $\left\{c_{n}\right\}$ be a sequence of positive numbers such that $c_{n}<1$ and

$$
\sum_{n=1}^{\infty} c_{n} /\left(1-c_{n}\right)<\epsilon
$$

where $\epsilon>0$ is given, put $r_{n}=c_{n} R_{n}, V_{n}=J\left(a_{n}, r_{n}\right)$, let $K$ consist of the origin and the union of the $V_{n}$ 's, and let $D$ be the complement of $K$. Since every boundary point of every $V_{n}$ is an essential boundary point of $D$ and the set of essential boundary points is closed, the origin is an essential boundary point of $D$.

Let $f \in B(D)$, and suppose $|f(z)|<1, f(\infty)=0$. At almost all points of the circumference $C_{n}$ of each $V_{n}, f(z)$ has well-defined nontangential boundary values, so that the path of integration in the Cauchy integral formula can be moved to $C_{n}$. If we put

$$
f_{n}(z)=\frac{1}{2 \pi i} \int_{C_{n}} \frac{f(t)}{t-z} d t
$$

then it is easily verified that

$$
f(z)=\sum_{n=1}^{\infty} f_{n}(z),
$$

the series converging uniformly in every closed subset of $D$. Moreover, if $z \notin G_{n}$, where $G_{n}$ is the interior of $J\left(a_{n}, R_{n}\right)$, we have

$$
\left|f_{n}(z)\right| \leqq \frac{r_{n}}{R_{n}-r_{n}}=\frac{c_{n}}{1-c_{n}} .
$$

If $G=\bigcup G_{n}$, it follows that the series defining $f(z)$ converges absolutely and uniformly on the complement $H$ of $G$. Thus $f(z)$ is continuous on $H$, which includes the origin, and $|f(z)| \leqq \epsilon$ on $H$. 
If $L$ is now any arc in $H$, with one end point at the origin, then $\lim f(z)$ exists as $z \rightarrow 0$ along $L$, and does not exceed $\epsilon$ in absolute value. This is so even if the cluster set of $f$ at 0 consists of the entire unit disc.

\section{REFERENCES}

1. L. V. Ahlfors, Bounded analytic functions, Duke Math. J. vol. 14 (1947) pp. 1-11.

2. L. V. Ahlfors and Arne Beurling, Conformal invariants and function-theoretic null-sets, Acta Math. vol. 83 (1950) pp. 101-129.

3. Lipman Bers, On rings of analytic functions, Bull. Amer. Math. Soc. vol. 54 (1948) pp. 311-315.

UNIVERSITY OF ROCHESTER, ROCHESTER, N. Y. 